\title{
Recurrent lateral patella dislocation affects knee function as much as ACL deficiency - however patients wait five times longer for treatment
}

Truls Martin Straume-Næsheim ${ }^{1,2^{*}}{ }^{0}$, Per-Henrik Randsborg ${ }^{1}$, Jan Rune Mikaelsen ${ }^{1}$, Einar Andreas Sivertsen ${ }^{3}$, Brian Devitt ${ }^{4}$, Lars-Petter Granan ${ }^{5}$ and Asbjørn Årøen ${ }^{6,1}$

\begin{abstract}
Background: Surgical treatment of young patients with recurrent lateral patella dislocation (RLDP) is often recommended because of loss of knee function that compromises their level of activity or even their daily life functioning. This situation is comparable to young patients with an anterior cruciate ligament (ACL) rupture. The purpose of this study was therefore to explore the time from injury to surgery and the pre-operative symptoms and knee function of young RLPD patients scheduled for stabilizing surgery and compare this group to age and sex-matched ACL-deficient patients.

Method: Forty-seven patients with unilateral RLPD listed for isolated medial patellofemoral ligament reconstruction were included in the study (RLPD-group). This group was compared to an age, sex and BMI matched ACL patient group obtained from the Norwegian knee ligament registry (ACL-group) for the following outcome measures: the knee injury and osteoarthritis outcome score (KOOS) assessed on the day of surgery and time from injury to surgery.

Results: The RLPD-group scored significantly lower than the ACL-group for the three KOOS subscales "Pain" (73.6 vs. 79.8, $p<0.05)$, "Symptoms" (71.7 vs. 79.3, $p<0.05)$ and "ADL" (84.7 vs 89.5, $\mathrm{p}<0.05)$. The lowest KOOS values were found for Sports/Recreation (53.5 vs. $51.3, p=0.65$ ) and Quality of life (37.6 vs. 36.7, $p=0.81$ ). The average time from primary injury to surgery was 6 months for the ACL group and 31 months for the RLPD group.

Conclusion: RLPD affected knee function as much as ACL deficiency, and was associated with more pain. Still the RLDP patients waited on average 5 times longer for surgery.

Trial registration: The patients with RLPD consisted of patients who were examined for possible recruitment for a concurrent prospective randomized controlled trial comparing conservative treatment and isolated surgical medial patellofemoral ligament (MPFL) reconstruction (Clinical trials no: NCT02263807, October 2014).
\end{abstract}

Keywords: Patella dislocation, Young adults and adolescents, Symptoms and function evaluation, Indication for surgery, Time to surgery, Comparative study

\footnotetext{
* Correspondence: trul@ahus.no

${ }^{1}$ The Department of Orthopaedic Surgery, Akershus University Hospital, 1478

Lørenskog, Norway

${ }^{2}$ Oslo Sports Trauma Research Center, Department of Sports Medicine,

Norwegian School of Sport Sciences, Oslo, Norway

Full list of author information is available at the end of the article
}

(c) The Author(s). 2019 Open Access This article is distributed under the terms of the Creative Commons Attribution 4.0 International License (http://creativecommons.org/licenses/by/4.0/), which permits unrestricted use, distribution, and reproduction in any medium, provided you give appropriate credit to the original author(s) and the source, provide a link to the Creative Commons license, and indicate if changes were made. The Creative Commons Public Domain Dedication waiver (http://creativecommons.org/publicdomain/zero/1.0/) applies to the data made available in this article, unless otherwise stated. 


\section{Introduction}

Lateral patella dislocation is a common serious knee injury that occurs mostly among adolescents and young adults. Patients with lateral patella dislocation give reports of considerably reduced knee function, quality of life and pain compared to reference values for the same age group for many years after their primary event [1]. In addition, $17-42 \%$ are likely to experience further dislocations, which substantially increase the risk of developing subsequent instability problems, patellofemoral pain and decreased knee function $[1,2]$.

It is well-known that patients with patella dislocation form a heterogeneous group in terms of anatomic risk factors, dislocation frequency and injury mechanisms, and there is a frequent recurring discussion related to "who, when and how" in the treatment of these patients $[3,4]$. Nevertheless, there seems to be an agreement in the literature that patients with recurrent lateral patella dislocations (RLPD) should be considered for surgery [5]. A significant factor endorsing surgical treatment is that these young patients lose confidence in the injured knee and experience symptoms and loss of function that compromises their level of activity or even their daily life functioning [1]. Patella stabilising surgery, and isolated medial patella femoral ligament (MPFL) reconstruction in particular, has started to show promising results with a high number of young patients returning to sport after surgery, and low incidence of recurrent instability [6].

This situation is comparable to another common knee injury within the same age group, the anterior cruciate ligament (ACL) rupture. The current recommended indication in Scandinavia for ACL reconstruction is persistent instability of the knee and a subjective feeling of give away after 612 weeks of functional rehabilitation and/or an activity level in demand of a good knee function [7-11]. Hence, surgical treatment of both RLPD patients and ACL deficient patients is based on persistent instability and the level of deteriorated knee function in these young patients. To some degree this is irrespective of the anatomical cause and therefore it would be interesting to compare the pre-operative kneerelated symptoms and function between these two groups. Particularly since the time from injury to surgery reported for the RLPD patients seems to be significantly longer than what is known from the ACL register data [9, 12-17].

The purpose of this study was therefore to explore the time from injury to surgery and the pre-operative symptoms and knee function of young RLPD patients scheduled for stabilizing surgery and compare this group to age and sex-matched ACL-deficient patients booked for surgical reconstruction.

\section{Methods}

\section{Study design}

A cross-sectional study was performed using a pre-operative questionnaire to assess knee function and symptoms in young patients with RLPD and sex, age and BMI matched patients with ACL injury awaiting surgical reconstruction.

\section{Patients}

The study recruited patients referred to the orthopaedic outpatient clinic at Akershus University Hospital for recurrent patella dislocations between May 2010 and December 2015. The patients with RLPD consisted of patients who were examined for possible recruitment for a concurrent prospective randomized controlled trial (RCT) comparing conservative treatment and isolated surgical medial patellofemoral ligament (MPFL) reconstruction (Clinical trials no: NCT02263807). The inclusion criteria listed in Table 1 were therefore based on the recommended indication for isolated MPFL reconstruction $[5,18]$. Bilateral cases were excluded as the protocol for the prospective RCT included comparisons with the contralateral leg in the follow-ups. All patients eligible for isolated MPFL reconstruction were asked to fill in the baseline assessment forms on the day of surgery.

The ACL group were selected among patients with isolated primary ACL reconstruction (+/- meniscal procedures) registered in the Norwegian National Knee Ligament Registry (NKLR) from 2004 to 2012. The NKLR was established in June 2004 and all Norwegian hospitals performing ACL-reconstructions report to the registry with a compliance rate of approximately $80 \%$ [19]. The ACL patients were cross-matched with the

Table 1 Inclusion, exclusion and matching criteria for the patients with recurrent patella dislocation (RLPD group) and their ACL-deficient patients (ACL) in the study

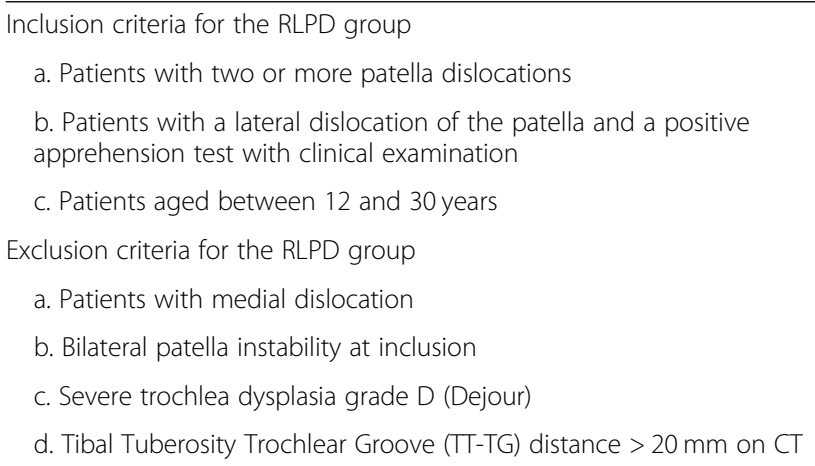


RLPD patients based on sex, age and BMI. Each RLPD case was matched with two ACL patients.

\section{Outcome measures}

The main outcome measure was time from primary injury to surgery and the Knee injury and Osteoarthritis Outcome Score (KOOS) filled in on the day of surgery by the the RLPD patients for this study and by the ACL patients as per protocol for the NKLR.

KOOS consists of 42 questions divided into 5 subscales; Pain, Symptoms, ADL (activities of daily living), Sport/Rec (function in sports and recreation) and QoL (knee related quality of life). Each question is scored from 0 (worst) to 4 (best) on a Likert scale, and a normalized score from 0 to 100 is calculated for each subscale which are independently used in all outcome comparisons, as recommended [20]. Calculation of the score of each subscale and missing data were treated according to the guidelines provided by Roos et al. [20].

The NKLR provides simple demographic data such as age, sex, height and weight, and corresponding data was also filled in pre-operatively by the RLPD patients. All RLPD patients also filled in their pre-injury Tegner activity score which is a one-item score from 0 to 10 where 0 represents disability due to the current knee condition and 10 represents competitive level in pivoting sports [21]. However, activity data was not registered in the NKLR for the ACL patients in the study period.

\section{Statistics}

Statistical analyses were performed using SPSS 22 (SPSS Inc., Chicago, Illinois). A paired sample T-test was chosen using the aggregated mean KOOS score for the two sex, age and BMI matched ACL controls as pair for each one of the RLPD patients. This was performed in consensus with previously published studies using NKLR data [22, 23 in order to rule out known confounders for the KOOS score such as age and sex [24-26]. An independent T-test or the Chi Squared test was used to compare the demographic data according to type and distribution of the examined variable. The level of significance for all tests was defined as $p<0.05$. Power analysis revealed that 18 pairs of patients were needed to detect a difference in KOOS QoL of 10 points (SD 15) with a power of 0.80 based on reference data from the developers of the KOOS [27].

\section{Results}

From May 2010 to December 2015, a total of 134 patients were referred to our outpatient clinic for suspected recurrent patella dislocations. As shown in Fig. 1,

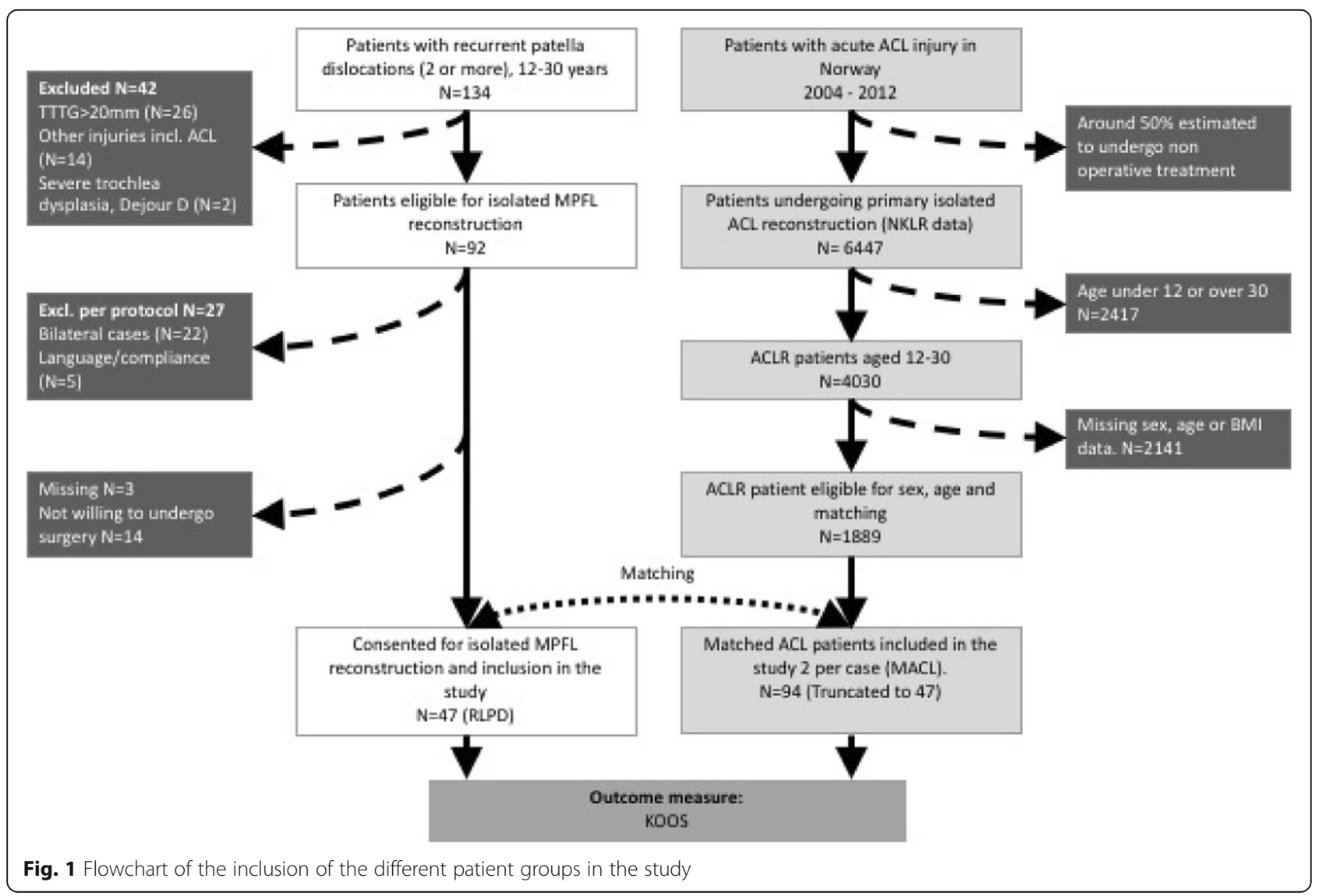


a total of 92 of these RLPD patients $(68.7 \%)$ were found eligible for treatment with isolated MPFL reconstruction. Notably, only two cases (1.5\%) were found to have a severe trochlear dysplasia as their main cause for RLPD and were referred for a trochleaplasty, and a total of 17 (12.7\%) did not want any surgical treatment. After exclusion of bilateral cases and patients not able to complete forms due to language barriers (the forms were only available in English and Norwegian), 47 (51.1\%) RLPD patients were finally included in the study. These 47 were matched with 94 ACL patients selected from the NKLR registry (Fig. 1). The demographics for both groups and all the ACL patients eligible for matching are presented in Table 2. For the RLPD group the mean Tegner activity score was 4.4 (SD 2.8) and 25 (53.2\%) patients reported that their latest dislocating event happened during sporting activities, where football $(N=9$, 19.1\%) was most frequent.

For the main outcome measure KOOS; the RLPDgroup scored significantly lower than the ACL-group for the three subscales "Pain", "Symptoms" and "ADL" (Fig. 2). The lowest KOOS values were found for the Sports/Recreation (Sports/Rec) and Quality of life (QoL), but here there was no significant difference between the two groups. However, median time in months from injury to operation was significantly longer for the RLPD group (31, quartiles 16-69) compared to the ACL-group (6 (quartiles 4-12).

\section{Discussion}

This study found that patients with RLPD struggle with their knee injury for many years before surgery is attempted. Their KOOS subscores for the Sports and QoL function were particularly low, but these were at the same level as their age and BMI matched patients with ACL-deficient knees scheduled for surgery. In addition, the RLPD patients report worse scores for pain, knee related symptoms and ADL function compared to their ACL peers. It is therefore interesting that the RLPD patients had waited close to 2.5 years on average before they were considered for surgery, compared to the ACL patients where $50 \%$ were operated within 6 months after the injury.
One can argue that this discrepancy in time from injury to treatment might only be representative for the practise at the study hospital or region where these RPLD patients were recruited, but other studies have reported comparable numbers with time from first injury to surgery ranging from 1 to 7 years on average [12-17]. One obvious explanation is that there is a need for at least one re-dislocation to be considered as a patient with RLPD, and the time between the first and second event can vary from weeks to many years. However, the highest incidence of re-dislocation is reported to be within the first months, but the curve does not flatten out until after 2 years [28]. In contrast, for the ACL deficient patients the indication for surgery is usually based on persistent instability of the knee and subjective feeling of give away after 6-12 weeks of functional rehabilitation [7-11]. Furthermore, while the treatment and rehabilitation of the ACL deficient knees are well documented, the adequate approach for RLPD is still under debate [3-6]. However, recent reviews have shown that $84 \%$ of the patients return to sport after stabilising surgery for RLPD and the corresponding incidence of recurrent instability and reoperations were low [6, 29]. These results are comparable to what has been reported for ACL-reconstruction [30]. The best results are found for the RLPD patients where an isolated MPFL reconstruction is considered as sufficient, which was the case for the RLPD patients in this current study. Still, surgeons seems be more eager to propose a surgical treatment for ACL deficient patients than for those with RLPD.

One reason for this is that an ACL injury is known as a common "sports" injury and surgeons could be tempted to consider ACL injured patients as a group of patients with a higher activity level and a higher demand to their knee function, which in turn would trigger a recommendation for surgical reconstruction [31]. On the other hand, a larger number of the ACL patients may be high performance athletes which could be part of the explanation. They are high maintenance and seek surgical solution quickly, their teams have insurance and support and this brings them to the operating table much quicker than the RLPD patients. This study also observed that the pre-injury level of activity for

Table 2 Demographics for the different patient groups in the study

\begin{tabular}{|c|c|c|c|}
\hline & $\begin{array}{l}\text { Recurrent lateral patella } \\
\text { dislocation }(N=47)\end{array}$ & $\begin{array}{l}\text { Matched } A C L \text {-deficient patients } \\
(A C L) \text { group }(N=94)\end{array}$ & $\begin{array}{l}\text { All eligible } A C L \text { patients from the } \\
A C L \text { registry }(N K L R)(N=1889)\end{array}$ \\
\hline Age at time of operation/inclusion (mean, (SD)) & $19.2(5.0)$ & $19.5(4.9)$ & $21.4(4.9)^{*}$ \\
\hline $\begin{array}{l}\text { Time in months from injury to operation (median, } \\
\text { (quartiles)) }\end{array}$ & $31(16-69)^{*}$ & $6(4-12)$ & $6(4-12)$ \\
\hline BMI (mean, (SD)) & $23.2(4.7)$ & $23.1(3.9)$ & $24.0(3.4)$ \\
\hline \multirow[t]{2}{*}{ Sex } & M: 15 (31.9\%) & M: 30 (31.9\%) & M: $894(47.3 \%)^{*}$ \\
\hline & F: $32(68.1 \%)$ & $F: 64(68.1 \%)$ & F: $995(52.7 \%)^{*}$ \\
\hline
\end{tabular}

*Significantly different from the other groups, $p<0.05$ 


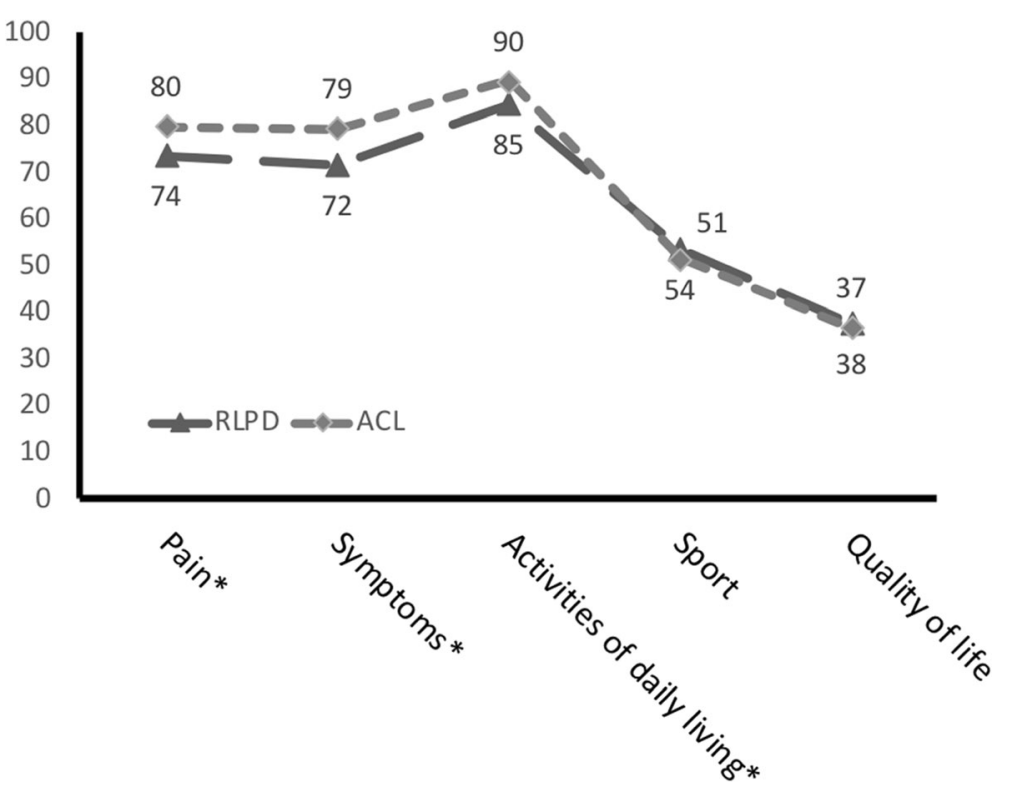

Fig. 2 Preoperative KOOS score for Recurrent lateral patella dislocation group (RLPD, $N=47)$ and the matched $A C L$ group $(A C L, N=94)$. ${ }^{*}$ Significantly different from RLDP, $p<0.05$

the RLPD patients was indeed lower compared to the numbers presented in the literature for patients with an ACL injury [32]. However, even though it seems like the RLPD patients do not have as high sporting demands as compared to the ACL deficient patients, more that $50 \%$ of the young RLPD patients reported that their last dislocation occurred during sports activities. Further on, they also report that their reduced knee function reduces their quality of life to the same level as for the patients with an ACL injury. In addition, they report more pain and everyday knee-related symptoms.

When comparing the KOOS scores for the RLPD group to previous studies assessing patients after a primary lateral patella dislocation, this study observed lower scores for all KOOS components. Interestingly, in the study by Magnussen et al. [1], they found no effect of re-dislocations on the KOOS. None of the cases in their study underwent surgical treatment; hence their result could be affected by a subgroup of RLPD patients that were coping quite well. In the inclusion process of this current study, 17 out of the 64 RLPD patients who were eligible for inclusion, felt that they were coping well with their knee injury or at least were not interested in surgical treatment.

\section{Limitations}

In contrast to most of the previous studies assessing symptoms and function in patients with RLPD [13, 3335], the RLPD patients included in this study were the ones eligible for treatment with isolated MPFL reconstruction. This limits the generalisability of the KOOS findings, but according to previous studies assessing the risk of further patella dislocations, these patients were likely to represent the milder spectrum of the RLPD patients [4].

The KOOS itself assesses impairments without a specific patellar instability component. However, KOOS has successfully been used in several studies assessing this patient group [1,36-38]. Further on, the main purpose of this study was not to assess the patellar instability, as all patients had known RLPD condition, but further to assess how well they were coping with this injury. In addition, the purpose was also to relate their impairments to a comparable group of ACL deficient patients, which would not be possible with a more patellofemoral-specific instrument. KOOS is a widely used and well-known instrument validated for assessing several different knee injuries and it has been reported to have the largest effect size in young active persons $[27,39]$.

\section{Perspectives}

The findings of this current study emphasise the importance to identify the RLPD patients who are not coping as early as possible, as a delayed treatment of these patients seems to result in a sustained inability to trust the knee as well as anxiety related to the fear of redislocation, which might prevent further participation in sports and recreational activities [2, 40]. Accordingly, a more thorough primary review of the patients with lateral patella dislocation is recommended in order to early identify those who are likely to re-dislocate, and further 
on to consider a routine follow-up (i.e. after 6-12 weeks) similar to the recommended follow-up routine which takes place after an isolated ACL rupture in Scandinavia $[7,8,31]$. Preferably, after a standardised rehabilitation programme focusing on stronger quadriceps (vastus medialis obliquus), stability training of the knee and hip, and protective bracing and/or taping to prevent early redislocations [3]. The goal must be to prevent that the patients with a primary lateral patella dislocation turn into RLPD patients and to identify the ones who do as early as possible.

\section{Conclusion}

Young patients with RLPD reported that their knee condition significantly reduced their sports function and quality of life to the same level as for their ACLdeficient peers. In addition, they experienced more pain, knee-related symptoms and restrictions in their activities of daily living. Still the RLDP patients wait on average 25 months longer for surgery.

\section{Abbreviations}

ACL: Anterior cruciate ligament; ADL: Activities of daily living; BMl: body mass index; KOOS: Knee injury and Osteoarthritis Outcome Score; MPFL: medial patellofemoral ligament; NKLR: Norwegian National Knee Ligament Registry; RCT: Randomized controlled trial; RLPD: recurrent lateral patella dislocation; TT-TG: Tibal Tuberosity Trochlear Groove

\section{Acknowledgements}

The authors would like to acknowledge all the colleagues at the Clinic of orthopaedic surgery, Akershus University Hospital for all help and patients in the lengthy inclusion process for this study.

\section{Ethics approval consent to participate}

The study protocol was approved by the Regional Committee of Medical and Health Research Ethics of South East Norway before initiation of the study (REC South East, reference 2009/2148). All RLPD patients provided oral and written consent to participate in the study. Participation in the NKLR is voluntary, both for surgeons and patients. All patients included in the NKLR have signed an informed consent that contains information about the NKLR, type of data recorded, data protection, follow-up procedure and possible participation in later research projects [19].

\section{Authors' contributions}

All authors contributed to study conception and design. TMSN, PHR and JRM have been the principal investigators of the study and were responsible for the data collection and TMSN performed the analyses, which were planned and checked by AA, EAS and LPG. LPG was responsible for the selection of $A C L$ patients. TMSN, PHR, AA, and BD wrote the first draft of the paper, and all authors contributed to the final manuscript. All authors read and approved the final manuscript.

\section{Funding}

The study has been supported by research grants from Sophies Minde Orthopaedics, Aase Bye and Trygve J. B. Hoff's Research Fund, The Norwegian Association of Sports Medicine, Research Grant and. South-Eastern Norway Regional Health Authority (grant no. 2016094).

\section{Availability of data and materials}

The datasets used and analysed for this current study are available from the corresponding author on reasonable request.

\section{Consent for publication}

Not applicable.

\section{Competing interests}

The authors declare that they have no competing interests.

\section{Author details}

${ }^{1}$ The Department of Orthopaedic Surgery, Akershus University Hospital, 1478 Lørenskog, Norway. ${ }^{2}$ Oslo Sports Trauma Research Center, Department of Sports Medicine, Norwegian School of Sport Sciences, Oslo, Norway. ${ }^{3}$ Department of Orthopaedic Surgery, Lovisenberg Diaconal Hospital, Oslo, Norway. ${ }^{4}$ OrthoSport Victoria, Melbourne, Australia. ${ }^{5}$ Oslo University Hospital, Oslo, Norway. ${ }^{6}$ Institute of Clinical Medicine University of Oslo, Campus Ahus. Clinic of Orthopaedic Surgery, Akershus University Hospital, Nordbyhagen, Norway.

Received: 1 November 2018 Accepted: 23 June 2019

Published online: 08 July 2019

\section{References}

1. Magnussen RA, Verlage M, Stock E, Zurek L, Flanigan DC, Tompkins M, et al. Primary patellar dislocations without surgical stabilization or recurrence: how well are these patients really doing? Knee Surg Sports Traumatol Arthrosc. 2015;25:2352-6.

2. Mäenpää H, Lehto MUK. Patellofemoral osteoarthritis after patellar dislocation. Clin Orthop Relat Res. 1997;339:156-62.

3. Smith TO, Donell S, Song F, Hing CB. Surgical versus non-surgical interventions for treating patellar dislocation. The Cochrane database of systematic reviews. 2015;2:Cd008106.

4. Balcarek P, Oberthur S, Hopfensitz S, Frosch S, Walde TA, Wachowski MM, et al. Which patellae are likely to redislocate? Knee Surg Sports Traumatol Arthrosc. 2014;22(10):2308-14.

5. Weber AE, Nathani A, Dines JS, Allen AA, Shubin-Stein BE, Arendt EA, et al. An algorithmic approach to the Management of Recurrent Lateral Patellar Dislocation. J Bone Joint Surg. 2016;98(5):417-27.

6. Schneider DK, Grawe B, Magnussen RA, Ceasar A, Parikh SN, Wall EJ, et al. Outcomes After Isolated Medial Patellofemoral Ligament Reconstruction for the Treatment of Recurrent Lateral Patellar Dislocations: A Systematic Review and Meta-analysis. Am J Sports Med. 2016;44:2993-3005.

7. Eitzen I, Moksnes H, Snyder-Mackler L, Risberg MA. A progressive 5-week exercise therapy program leads to significant improvement in knee function early after anterior cruciate ligament injury. J Orthop Sports Phys Ther. 2010; 40(11):705-21.

8. Grindem H, Granan LP, Risberg MA, Engebretsen L, Snyder-Mackler L, Eitzen I. How does a combined preoperative and postoperative rehabilitation programme influence the outcome of ACL reconstruction 2 years after surgery? A comparison between patients in the Delaware-Oslo ACL Cohort and the Norwegian National Knee Ligament Registry. Br J Sports Med. 2014; 49:385-9.

9. Ahldén M, Samuelsson K, Sernert N, Forssblad M, Karlsson J, Kartus J. The Swedish National Anterior Cruciate Ligament Register: A Report on Baseline Variables and Outcomes of Surgery for Almost 18,000 Patients. Am J Sports Med. 2012:40(10):2230-5.

10. Frobell RB, Roos EM, Roos HP, Ranstam J, Lohmander LS. A randomized trial of treatment for acute anterior cruciate ligament tears. N Engl J Med. 2010; 363(4):331-42.

11. Meuffels DE, Poldervaart MT, Diercks RL, Fievez AW, Patt TW, Hart CP, et al. Guideline on anterior cruciate ligament injury. Acta Orthop. 2012; 83(4):379-86.

12. Camp CL, Krych AJ, Dahm DL, Levy BA, Stuart MJ. Medial patellofemoral ligament repair for recurrent patellar dislocation. Am J Sports Med. 2010; 38(11):2248-54.

13. Christiansen SE, Jacobsen BW, Lund B, Lind M. Reconstruction of the medial patellofemoral ligament with gracilis tendon autograft in transverse patellar drill holes. Arthroscopy. 2008;24(1):82-7.

14. Drez D Jr, Edwards TB, Williams CS. Results of medial patellofemoral ligament reconstruction in the treatment of patellar dislocation. Arthroscopy. 2001;17(3):298-306.

15. Ellera Gomes JL. Medial patellofemoral ligament reconstruction for recurrent dislocation of the patella: a preliminary report. Arthroscopy. 1992;8(3):335-40.

16. Lim AK, Chang HC, Hui JH. Recurrent patellar dislocation: reappraising our approach to surgery. Ann Acad Med Singap. 2008;37(4):320-3.

17. Slenker NR, Tucker BS, Pepe MD, Marchetto PA, Cohen SB. Short -/intermediate-term outcomes after medial patellofemoral ligament 
reconstruction in the treatment of chronic lateral patellofemoral instability Phys Sportsmed. 2013:41(2):26-33.

18. Kita K, Tanaka Y, Toritsuka Y, Amano H, Uchida R, Takao R, et al. Factors affecting the outcomes of double-bundle medial patellofemoral ligament reconstruction for recurrent patellar dislocations evaluated by multivariate analysis. Am J Sports Med. 2015.

19. Granan LP, Bahr R, Steindal K, Furnes O, Engebretsen L. Development of a national cruciate ligament surgery registry: the Norwegian National Knee Ligament Registry. Am J Sports Med. 2008;36(2):308-15.

20. Roos EM, Roos HP, Lohmander LS, Ekdahl C, Beynnon BD. Knee injury and osteoarthritis outcome score (KOOS)--development of a self-administered outcome measure. J Orthop Sports Phys Ther. 1998;28(2):88-96.

21. Tegner Y, Lysholm J. Rating systems in the evaluation of knee ligament injuries. Clin Orthop Relat Res. 1985;(198):43-9.

22. Hjermundrud V, Bjune TK, Risberg MA, Engebretsen L, Aroen A. Fullthickness cartilage lesion do not affect knee function in patients with $\mathrm{ACL}$ injury. Knee Surg Sports Traumatol Arthrosc. 2010;18(3):298-303.

23. Rotterud JH, Risberg MA, Engebretsen L, Aroen A. Patients with focal fullthickness cartilage lesions benefit less from ACL reconstruction at 2-5 years follow-up. Knee Surg Sports Traumatol Arthrosc. 2012;20(8):1533-9.

24. Ageberg E, Forssblad M, Herbertsson P, Roos EM. Sex Differences in PatientReported Outcomes After Anterior Cruciate Ligament Reconstruction: Data From the Swedish Knee Ligament Register. Am J Sports Med. 2010;38(7): 1334-42.

25. Cameron KL, Thompson BS, Peck KY, Owens BD, Marshall SW, Svoboda SJ. Normative values for the KOOS and WOMAC in a young athletic population: history of knee ligament injury is associated with lower scores. Am J Sports Med. 2013:41(3):582-9.

26. Paradowski PT, Bergman S, Sunden-Lundius A, Lohmander LS, Roos EM. Knee complaints vary with age and gender in the adult population. Population-based reference data for the knee injury and osteoarthritis outcome score (KOOS). BMC Musculoskelet Disord. 2006;7:38.

27. Roos E, Lohmander LS. The knee injury and osteoarthritis outcome score (KOOS): from joint injury to osteoarthritis. Health Qual Life Outcomes. 2003; 1(1):64.

28. Palmu S, Kallio PE, Donell ST, Helenius I, Nietosvaara Y. Acute patellar dislocation in children and adolescents: a randomized clinical trial. J Bone Joint Surg Am. 2008;90(3):463-70.

29. Sherman SL, Deasis DP, Garrone AJ, Voss EE, Oliver HA. Return to play after patellar stabilization. Curr Rev Musculoskelet Med. 2018;11(2):280-4.

30. Ardern CL, Taylor NF, Feller JA, Webster KE. Fifty-five per cent return to competitive sport following anterior cruciate ligament reconstruction surgery: an updated systematic review and meta-analysis including aspects of physical functioning and contextual factors. Br J Sports Med. 2014;48(21): 1543-52.

31. Eitzen I, Moksnes H, Snyder-Mackler L, Engebretsen L, Risberg MA. Functional tests should be accentuated more in the decision for ACL reconstruction. Knee Surg Sports Traumatol Arthrosc. 2010;18(11):1517-25.

32. Eggerding V, Meuffels DE, Bierma-Zeinstra SM, Verhaar JA, Reijman M. Factors related to the need for surgical reconstruction after anterior cruciate ligament rupture: a systematic review of the literature. J Orthop Sports Phys Ther. 2015;45(1):37-44.

33. Nomura E, Inoue M. Hybrid medial patellofemoral ligament reconstruction using the semitendinous tendon for recurrent patellar dislocation: minimum 3 years' follow-up. Arthroscopy. 2006;22(7):787-93.

34. Nomura E, Inoue M, Kobayashi S. Long-term follow-up and knee osteoarthritis change after medial patellofemoral ligament reconstruction for recurrent patellar dislocation. Am J Sports Med. 2007;35(11):1851-8.

35. Schottle PB, Fucentese SF, Romero J. Clinical and radiological outcome of medial patellofemoral ligament reconstruction with a semitendinosus autograft for patella instability. Knee Surg Sports Traumatol Arthrosc. 2005; 13(7):516-21.

36. Christiansen SE, Jakobsen BW, Lund B, Lind M. Isolated repair of the medial patellofemoral ligament in primary dislocation of the patella: a prospective randomized study. Arthroscopy. 2008;24(8):881-7.

37. Arendt E, Agel J, Moore A. First time lateral patella dislocations: characterizing their readiness for return to activity. Br J Sports Med. 2011; 45(4):335-6.

38. Witonski D, Keska R, Synder M, Sibinski M. An isolated medial patellofemoral ligament reconstruction with patellar tendon autograft. Biomed Res Int. 2013;2013:637678
39. Wang $\mathrm{D}$, Jones $\mathrm{MH}$, Khair MM, Miniaci A. Patient-reported outcome measures for the knee. J Knee Surg. 2010;23(3):137-51.

40. Magnussen RA, Trojani C, Granan LP, Neyret P, Colombet P, Engebretsen L, et al. Patient demographics and surgical characteristics in ACL revision: a comparison of French, Norwegian, and North American cohorts. Knee Surg Sports Traumatol Arthrosc. 2015;23(8):2339-48.

\section{Publisher's Note}

Springer Nature remains neutral with regard to jurisdictional claims in published maps and institutional affiliations.
Ready to submit your research? Choose BMC and benefit from:

- fast, convenient online submission

- thorough peer review by experienced researchers in your field

- rapid publication on acceptance

- support for research data, including large and complex data types

- gold Open Access which fosters wider collaboration and increased citations

- maximum visibility for your research: over $100 \mathrm{M}$ website views per year

At $\mathrm{BMC}$, research is always in progress.

Learn more biomedcentral.com/submissions 